\title{
Molecular dynamics simulations and free energy profile of Paracetamol in DPPC and DMPC lipid bilayers
}

\author{
YOUSEF NADEMI ${ }^{\mathrm{a}}$, SEPIDEH AMJAD IRANAGH ${ }^{\mathrm{b}}$, ABBAS YOUSEFPOUR $^{\mathrm{a}}$, \\ SEYEDEH ZAHRA MOUSAVI ${ }^{\mathrm{a}}$ and HAMID MODARRESS ${ }^{\mathrm{a}, *}$ \\ ${ }^{\mathrm{a} D e p a r t m e n t ~ o f ~ C h e m i c a l ~ E n g i n e e r i n g, ~}{ }^{\mathrm{b}}$ Department of Chemistry, Amirkabir University of Technology, \\ 15875-4413, Tehran, Iran \\ e-mail: hmodares@aut.ac.ir
}

MS received 1 July 2013; revised 21 September 2013; accepted 14 October 2013

\begin{abstract}
Molecular dynamics (MD) simulations and biased MD simulation were carried out for the neutral form of Paracetamol inserted in fully hydrated dipalmitoylphosphatidylcholine (DPPC) and dimyristoylphosphatidylcholine (DMPC) lipid bilayers. For comparison, fully hydrated DMPC and DPPC lipid bilayers were also simulated separately without Paracetamol. The simulation time for each system was 50 ns. At two concentrations of Paracetamol, various properties of the lipid bilayer such as area per lipid, order parameter, diffusion coefficient, radial distribution function, electrostatic potential, mass density and hydrogen bonds have been calculated. Also, the convergence in time of the free energy profile of the Paracetamol along a DPPC bilayer normal was calculated by umbrella sampling method. From the obtained results, it can be concluded that neutral form of Paracetamol shows a generally similar behaviour in DPPC and DMPC lipid bilayers. It was shown that the addition of Paracetamol causes a decrease in tail order parameter of both DPPC and DMPC lipid bilayers and the tail of Paracetamol adopts an inward orientation in the lipid bilayers. Also from the free energy profile, the high penetration barrier in the bilayer centre was determined.
\end{abstract}

Keywords. Lipid bilayers; Paracetamol; free energy; molecular dynamics simulation; membrane.

\section{Introduction}

Drugs often interact with cell membranes in their pathway of final action. Equilibrium position of specific drugs in target membranes also affects their metabolism and transport. ${ }^{1}$ The most important membrane for drug administration is the plasma membrane, through which drugs must penetrate to reach the internal milieu of the target cells. ${ }^{2}$ Among various related factors that govern the behaviour of drugs in the cell membranes; shape, size, hydrophobicity and $p K_{a}$ are the most influential. ${ }^{3}$ Understanding the underlying interaction between the drug and lipid is important for improving the efficacy and viability of many drugs. The most abundant lipids in mammalian membranes are phosphatidylcholines (PC) $;{ }^{4}$ and therefore PC bilayers are commonly used as simple membrane models.

In this study, the free energy profile of Paracetamol in dipalmitoylphosphatidylcholine (DPPC) lipid bilayer and the interaction between Paracetamol (acetaminophen) and phospholipid membranes by using molecular

\footnotetext{
*For correspondence
}

dynamics (MD) simulation and biased MD simulation are investigated.

Paracetamol has been used as an analgesic and antipyretic agent since $1950 .{ }^{5}$ In combination with opioid analgesics, Paracetamol can also be used in the management of more severe pain such as post-surgical pain and providing palliative care in advanced cancer patients. ${ }^{6}$ Compared to other non-steroidal analgesic, it has an enviable safety profile, and apart from wellknown dose-dependent hepatotoxicity, it is generally considered a safe drug. ${ }^{7}$ It has a plasma half-life of around $2 \mathrm{~h}^{8}$ Paracetamol, as shown in figure 1(a), consists of a benzene ring core, substituted by one hydroxyl group and the nitrogen atom of an amide group in the Para $(1,4)$ pattern. ${ }^{9}$ It has a $p K_{a}$ value of 9.5 and is therefore largely unionized over the physiological range of $\mathrm{pH} .{ }^{10}$ It was proposed that Paracetamol exerted its analgesic action by inhibition of centrally situated isoforms of the cyclooxygenase enzyme. ${ }^{11}$ The concept of Paracetamol as a centrally acting cyclooxygenase inhibitor is probably incomplete, and the true mechanism of analgesia may be far more complex. ${ }^{12}$ Metabolism of the drug is primarily in the liver, via conjugation with glucuronic $(60 \%)$ and sulphuric 
$(35 \%)$ acids, or cysteine (3\%). ${ }^{13}$ To bring molecularlevel insight into the interaction between Paracetamol molecules and liver, it is imperative to understand in detail the thermodynamics and transport properties of Paracetamol with lipid membranes. Molecular dynamics simulations may provide valuable complementary to experiments information about details of interactions between the molecules. ${ }^{3,14}$ However, despite enormous growth of the number of studies on computer simulations of lipid bilayers and utilizing GROMACS package for MD simulations (for example, see recent reviews, 15-18) there are no investigations on computer modelling of analgesics in lipid bilayers. Also the interaction between Paracetamol (acetaminophen) and two model membranes made of DPPC and dimyristoylphosphatidylcholine (DMPC) molecules in their liquid crystalline states has been probed. To the best of our knowledge, there are no published results on molecular dynamics simulations of Paracetamol in a lipid bilayer. Therefore, the aims of this study can be expressed as follows: First, various characteristics of the behaviour of the neutral forms of Paracetamol in a model lipid membrane such as Paracetamol preferential localization, hydrogen bond formation, diffusion, etc. will be examined. Second, the effect of Paracetamol on different membrane properties will be investigated. Third, the mean position of Paracetamol in DPPC lipid bilayer is identified from calculation of free energy profile by using umbrella sampling method.

\section{Methodology}

\subsection{Molecular structures}

Simulations were performed on six different simulation systems. Three of these systems contained 128 DPPC lipids, which were hydrated by 3871 water molecules, and the next three simulation systems contained 128 DMPC lipids, which were hydrated by 3846 water molecules arranged in a bilayer with 64 lipids in each leaflet. Four of these simulation systems contained 4 and 8 neutral molecules of Paracetamol. Two systems which contained only lipid molecules and water were used as reference. Molecular structures of DPPC and DMPC lipids as well as neutral Paracetamol molecule are shown in figure 1 ( $a, b$ and $c)$. For lipid bilayers and Paracetamol molecules, the united atom model based on the GROMOS force field with modification by Berger was used. ${ }^{19,20}$ This force field has been validated previously by other researchers. ${ }^{21-23}$ For water molecules, the simple point charge (SPC) model was applied. ${ }^{24}$ The topology and coordinates file of Paracetamol based on the GROMOS87 force field was obtained from PRODRG server. ${ }^{25}$

\subsection{Simulation details}

Simulations were carried out in the NPT (an ensemble with constant Number of particles, Pressure and Temperature) ensemble using GROMACS4.5.2. ${ }^{26}$ The temperature was kept constant at $323 \mathrm{~K}$ and $310 \mathrm{~K}$, respectively for DPPC and DMPC by coupling the simulations cell to a velocity rescale (v-rescale) thermostat with a coupling time of $0.1 \mathrm{ps}^{27}$ All the bonds were kept constant with LINCS algorithm. ${ }^{28}$ The pressure was kept constant at 1 bar by a compressibility of $4.5 \times 10^{-5} \mathrm{bar}^{-1}$ and by coupling the simulations cell to a Parrinello-Rahman barostat, with a coupling time constant of $5.0 \mathrm{ps,}$, which was applied semiisotropically with two degree of freedom, one in the $Z$ direction and another in the $X Y$ direction. ${ }^{29}$ The integration step was 2 fs. Non-bonded interactions were calculated using a cutoff of $12 \AA$. Long-range electrostatic interactions are important for correct description of the lipid bilayer, and they were calculated by Particle Mesh Ewald summation (PME) algorithm, which was updated every 10th time step with grid spacing of $1.2 \AA$ and by cubic interpolation. ${ }^{30}$ The eventual centre of mass motion of the system was removed every 100 steps. The initial structure for DPPC lipid bilayer was taken from the biocomputing group at the University of Calgary (http://moose.bio.ucalgary.ca/). The drug molecules were added in equal amount (two and four drug molecules in the simulation systems) to each side of the bilayer and then, water molecules were added by using the tools included in the GROMACS package. Steepest descent energy minimization was used to remove bad contacts and settle down the water molecules within the system. ${ }^{31}$ All simulations were run for $50 \mathrm{~ns}$ from their initial conditions and the coordinates of the atoms were stored each picosecond for further analysis. For free energy calculations, in the biased MD simulation, pre-equilibrated simulation starting frames were generated. The starting frames for biased MD simulation were obtained by pulling the centre-of-mass $(\mathrm{COM})$ of Paracetamol against the COM of DPPC lipid bilayer. Paracetamol was pulled along the bilayer normal (the $z$-axis) by using a pulling force constant of $500 \mathrm{KJ} \mathrm{mol}^{-1} \mathrm{~nm}^{-2}$ and pulling rate of $0.03 \mathrm{~nm} \mathrm{ps}{ }^{-1}$. Pulling exerts a harmonic potential on the drug molecule and moves the potential centre with a given pull rate. From the pulling simulation, the snapshots were obtained and among them 16 windows ( $\sim 0.2 \AA$ apart) along the $z$-axis, ranging from the bulk 
(a)
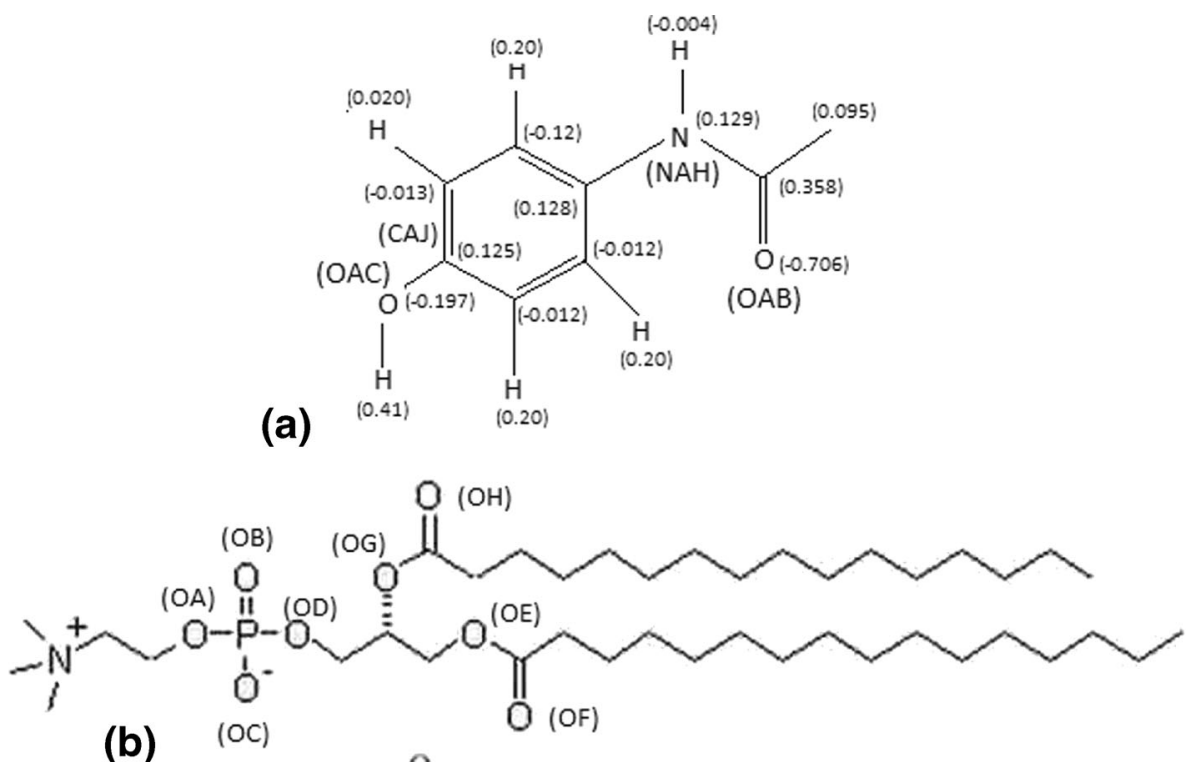

(b)

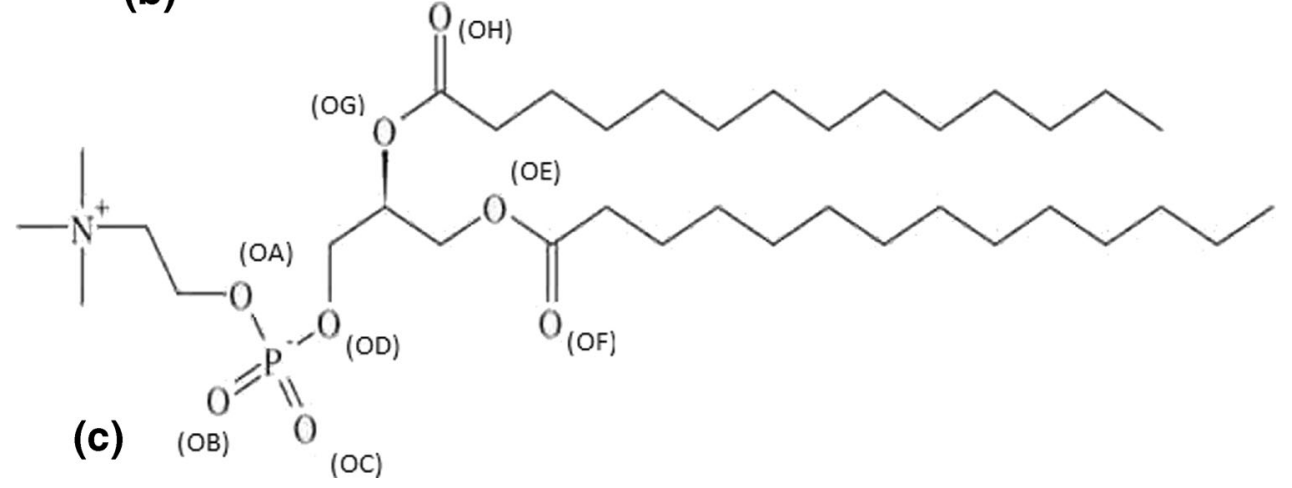

Figure 1. Molecular structure of Paracetamol molecule with partial atom charges and atom names referred in the text (a), DPPC lipid (b) and DMPC lipid (c).

water to the middle of the DPPC lipid bilayer, were selected. Each window was explored in a separate simulation (10 ns run time each) to avoid any drug interactions. The two monolayers in the lipid bilayer membrane were assumed to be symmetric and the potential of mean force (PMF) was calculated for one of them and then applied to the other one.

\section{Result and discussion}

\subsection{Mass density}

The calculated mass densities of the neutral Paracetamol, water and DPPC molecules for the simulation systems are shown in figure 2. Results indicate that Paracetamol molecules are located inside the bilayer under the lipid head groups in a similar manner as previously reported for alcohols. ${ }^{16,20}$ Paracetamol molecules have maximum density at a distance of $21 \AA$ from the bilayer centre in the area where the lipid head groups and esters are located. Similar effects are observed for systems with the DMPC lipid bilayer (see supplementary data S1). An important result is that; DPPC lipids in the reference system have higher maximum density comparing to its density in the systems containing Paracetamol molecules.

\subsection{Order parameter}

The lipid tails in the bilayer membrane are highly dynamic and many movements on different time scales can take place such as rotation around chemical bonds and trans/gauche isomerization, rotation around lipid axis, lateral diffusion and some other motions. ${ }^{16}$ The order parameter value of the lipid tails can be used to study the chain mobility in the lipid membrane systems. ${ }^{32}$ Lipid acyl chain order parameters are obtained easily from deuterium NMR quadrupole splittings as well as simulations. ${ }^{16}$ The orientation and ordering of lipid molecules in a bilayer are commonly represented by using the C-deuterium order parameter, which is determined by the tensor $S$ in the following 


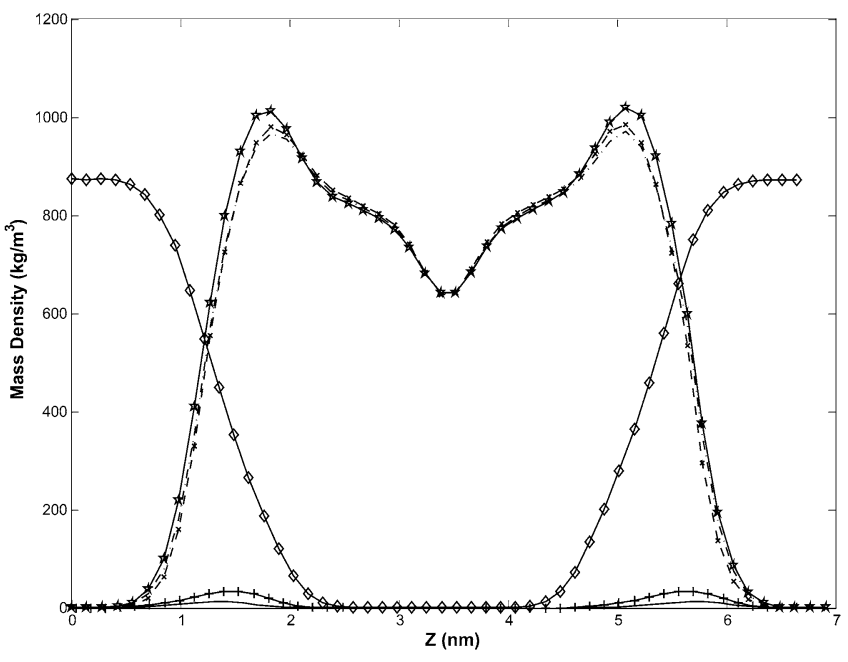

Figure 2. Mass density of different components for DPPC lipid bilayer systems: 4 Neutral Paracetamol system (-), 8 Neutral Paracetamol system (+), DPPC reference system (文), DPPC system containing 4 Paracetamol $\left({ }^{\times}\right)$, DPPC system containing 8 Paracetamol (___), water $(\diamond)$.

equation: ${ }^{16}$

$$
S=\frac{1}{2}\left\langle 3 \cos ^{2} \theta-1\right\rangle,
$$

where $\theta$ is the angle formed between the $Z$ direction and the outward pointing normal of the bilayer. The angle brackets imply averaging over time and molecules. To calculate the orientation of Paracetamol in the lipid bilayer, the molecular vector is defined by two chosen atoms. The vector NAH-CAJ (see figure 1) can be associated with the Paracetamol long axis. Order parameters for carbons of sn 1 chain of DPPC lipids are shown in figure 3 (calculations for the sn2 chain shows similar result). Calculated values for the reference bilayer agree with the experimental and previous simulation results. ${ }^{20,33,34}$ Results in figure 3 show a decrease in the order parameter of DPPC, for the system containing neutral form of Paracetamol and also the same effects have been observed in DMPC lipid bilayers. It has been shown that various inclusions into the membrane have different effects on the order parameters of the lipid tails. ${ }^{14}$ For example, it is proven that, both from molecular dynamic simulations and experiments that the addition of cholesterol leads to an increase in the order parameters, and addition of the local anesthetics benzocaine and charged articaine causes a decrease of the order parameters in lipid bilayers. ${ }^{14,16,35}$ An increase or decrease of the order parameters do not necessarily represent the trend of change in the membrane fluidity. The reason can be explained by considering the fact that addition of some drugs such as the neutral form of articaine, which increases the order parameter of the lipid bilayers leads to a faster diffusion of the lipids. ${ }^{14}$
Results of this study indicate that the addition of neutral form of Paracetamol decreases the order parameter and leads to slower diffusion of the lipid molecules (see table 1). Angular distribution of the vector angle $\Phi$ for the long axis (the axis NAH-CAJ in figure 1a) of Paracetamol in DPPC lipid bilayer membrane is shown in figure 4 , where the zero angle $(\Phi=0)$ corresponds to the direction outward of the bilayer. From figure 4 it is seen that the most probable angle is $\Phi=103.2^{\circ}$ in the DPPC lipid bilayer and $\Phi=104.1^{\circ}$ in the DMPC lipid bilayer (see supplementary data S2), which indicates preferential inward pointing of the NAH-CAJ vector in the lipid bilayers.

\subsection{Hydrogen bonds}

Hydrogen bonds can be formed between Paracetamol molecules with water and different polar head groups of the lipid as well as between lipids and water. The presence of hydrogen bonds was determined with a maximum distance of $2.5 \AA$ between acceptor and hydrogen and with the maximum angle of $30^{\circ}$ between the acceptor-hydrogen and donor -hydrogen vectors. ${ }^{36,37}$ Here, several hydrogen bonding forms have been explored: between Paracetamol molecules and the lipids, between water and the lipids as well as between water and Paracetamol. Results are summarized in table 2 .

Results show that Paracetamol molecules form almost the same number of hydrogen bonds with DMPC lipids and DPPC lipids (see supplementary data S3). Hydrogen bonding between water molecules and Paracetamol shows similar trends in presence of DMPC and DPPC lipid bilayers. Most of such hydrogen bonds are formed between carbonyl oxygen $(\mathrm{OAB})$ of the drug molecules (see figure 1a) and water; also a few hydrogen bonds were formed with other polar atoms such as OAC or NAH. Effect of Paracetamol on hydrogen bonding between the lipids and water is low, and no noticeable decrease is observed (see table 2).

\subsection{Area per lipid}

One of the important quantities in describing the behaviour of lipid bilayers is the average area per lipid. It provides a measure for tuning the force fields and other parameters for the lipid systems and is used for quantitative description of lipid bilayer behaviour by MD simulations. In this study, the average area per lipid molecule was calculated by multiplying the $X Y$ dimensions of the simulation box and dividing the result by the number 

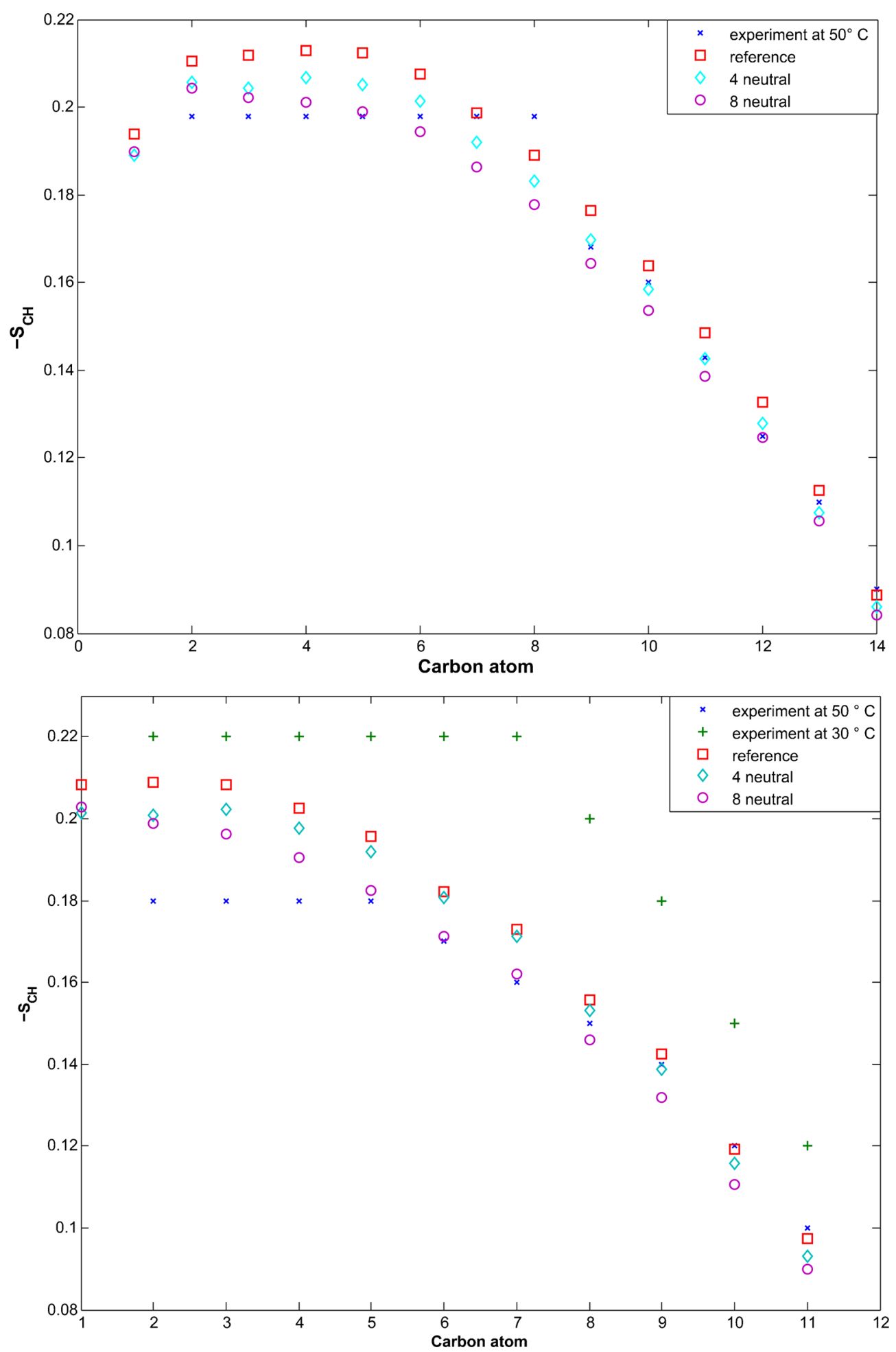

Figure 3. $\mathrm{CH}$ bond order parameters for different carbon atoms in the sn 1 chain of lipids. DPPC (top) and DMPC. ${ }^{33}$

of lipid molecules present in one leaflet of the bilayer. The average area per lipid for different simulations are shown in table 1 . Results show no drift in evolution of the area per lipid for different simulated systems, and therefore, the systems were considered to be in equilibrium. From table 1, the calculated area per 
Table 1. Important quantitative properties of the simulated bilayer systems: Area per lipid; $D_{\mathrm{A}}$ Paracetamol lateral diffusion coefficient; $D_{\mathrm{L}}$ lipid lateral diffusion coefficient; $\omega_{\text {mid }}$ electrostatic potential in the middle of membrane; $\omega_{\max }$ maximum electrostatic potential in the membrane.

\begin{tabular}{lcccccc}
\hline System & $\begin{array}{c}\text { DPPC in } \\
\text { presence of } 4 \\
\text { neutral drug }\end{array}$ & $\begin{array}{c}\text { DPPC in } \\
\text { presence of } 8 \\
\text { neutral drug }\end{array}$ & $\begin{array}{c}\text { DPPC reference } \\
\text { (without drug) }\end{array}$ & $\begin{array}{c}\text { DMPC in } \\
\text { presence of 4 } \\
\text { neutral drug }\end{array}$ & $\begin{array}{c}\text { DMPC in } \\
\text { presence of } 8 \\
\text { neutral drug }\end{array}$ & $\begin{array}{c}\text { DMPC reference } \\
\text { (without drug) }\end{array}$ \\
\hline$A\left(\AA^{2}\right)$ & 64.96 & 65.55 & 64.20 & 61.25 & 61.80 & 61.03 \\
$D_{\mathrm{A}}\left(10^{8} \frac{\mathrm{cm}^{2}}{\mathrm{~s}}\right)$ & 64.10 & 108.70 & - & 17.90 & 33.20 & - \\
$D_{\mathrm{A}}\left(10^{8} \frac{\mathrm{cm}^{2}}{\mathrm{~s}}\right)$ & 8.30 & 7.90 & 23.80 & 5.60 & 4.80 & 18.60 \\
$\omega_{\operatorname{mid}}(V)$ & 0.58 & 0.61 & 0.58 & 0.54 & 0.57 & 0.56 \\
$\omega_{\max }(V)$ & 0.78 & 0.79 & 0.80 & 0.75 & 0.77 & 0.82 \\
\hline
\end{tabular}

$\mathrm{V}=$ volt

lipid of DPPC reference system is $64.20 \AA^{2}$, which is close to the experimental value of $62 \AA^{2}$ at $323 \mathrm{~K}$ and $1 \mathrm{bar}$, and is in agreement with the values obtained in previous simulations. ${ }^{38-44}$ The experimental value of area per lipid for DMPC at $297 \mathrm{~K}$ and 1 bar is $60.6 \AA^{2},{ }^{44}$ and is in close agreement with the calculated value of $61.03 \AA^{2}$ in this study. The area per lipid which is in the range of $61-67 \AA^{2}$ corresponds to the liquid crystalline phase. ${ }^{14}$

Results show that both membranes in presence of the drug molecules have larger area per lipid compared with the reference system. The reason can be explained by the fact that the drug molecules occupy some spaces between the lipid molecules and thereby increase the average area per lipid.

\subsection{Radial distribution function}

In order to obtain a better insight into the coordination, hydration, and probability distribution of water molecules around the lipid bilayer, and to examine the effect of the drug on these parameters, the radial distribution function (RDF) analysis has been utilized. Figure 5 displays the RDF of oxygen atom in water around oxygen atom in phosphate lipid bilayers. All the RDFs, presented in figure 5 display their first minimum at about $3.2 \AA$, which shows the hydrogen bonding of water to phosphate oxygen, and also display the second maximum at about $5 \AA$. It is seen from this figure that the systems containing neutral drugs have somewhat higher RDF compared to the reference systems. By integration of the RDFs up to the first minimum, the coordination numbers are evaluated and presented in table $3 .{ }^{14}$ These results can be explained by considering the area per lipid in the systems containing Paracetamol, which show an increase due to its orientation in the lipid bilayers and as a result more space is created in the lipid bilayers to allow water molecules to enter into the lipid bilayers.

\subsection{Lateral diffusion}

Diffusion is one of the important dynamical properties of the lipid bilayer. ${ }^{14}$ Different membrane parameters can be controlled by the lateral motion of lipids. For instance, Venable et al. proposed that there is an intimate connection between the microviscosity of the head group region and upper hydrophobic part of the phospholipids, and the lateral diffusion rate. ${ }^{41}$ In this study, the lateral dffusion coefficient of Paracetamol molecules and lipids were calculated by using Einstein's relation based on the mean squared displacement (MSD) of the molecules over the course of the simulation. Einstein's equation in two dimensions is in the following form: ${ }^{14}$

$$
D=\lim _{t \rightarrow \infty} \frac{1}{4} \frac{d}{d t}\left\langle|\Delta \mathbf{r}(t)|^{2}\right\rangle,
$$

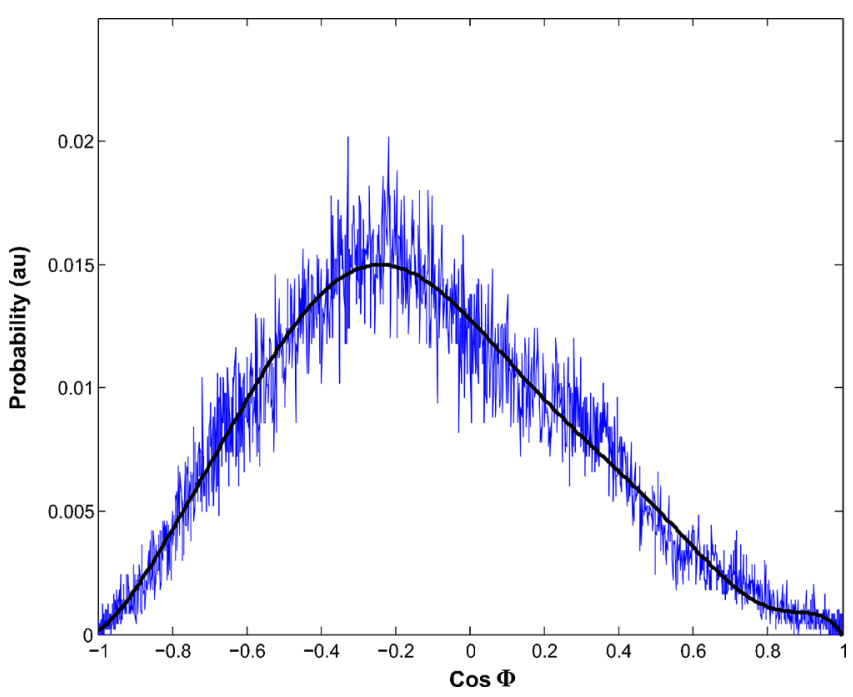

Figure 4. Angular distribution of the Paracetamol long axis in DPPC lipid bilayer. 
Table 2. Average number of different hydrogen bonds in the simulated systems.

\begin{tabular}{lccccc}
\hline Simulation system & $\begin{array}{c}\text { Number } \\
\text { of drugs }\end{array}$ & $\begin{array}{c}\text { Hydrogen bonds } \\
\text { between: }\end{array}$ & $\begin{array}{c}\text { Total bonds } \\
\text { per time frame* }\end{array}$ & $\begin{array}{c}\text { Bonds per } \\
\text { Paracetamol }\end{array}$ & Bonds per lipid \\
\hline Drug+DPPC+water & 4 & Drug and DPPC & 0.584 & 0.146 & - \\
Drug+DPPC+water & 8 & Drug and DPPC & 1.326 & 0.166 & - \\
Drug+DPPC+water & 4 & Drug and water & 8.772 & 2.193 & - \\
Drug+DPPC+water & 8 & Drug and water & 16.637 & 2.080 & - \\
Drug+DPPC+water & 4 & DPPC and water & 788.073 & - & 6.157 \\
Drug+DPPC+water & 8 & DPPC and water & 787.178 & - & 6.150 \\
Drug+DPPC+water & 0 & DPPC and water & 789.211 & - & - \\
Drug+DMPC+water & 4 & Drug and DMPC & 0.795 & 0.199 & - \\
Drug+DMPC+water & 8 & Drug and DMPC & 1.312 & 0.164 & - \\
Drug+DMPC+water & 4 & Drug and water & 8.958 & 2.239 & - \\
Drug+DMPC+water & 8 & Drug and water & 17.320 & 2.165 & - \\
Drug+DMPC+water & 4 & DMPC and water & 789.617 & - & 6.169 \\
Drug+DMPC+water & 8 & DMPC and water & 788.215 & - & 6.181 \\
Drug+DMPC+water & 0 & DMPC and water & 791.121 & - & \\
\hline
\end{tabular}

$*$ Time frame $=$ data storage in $1 \mathrm{ps}$

where $\left\langle|\Delta \mathbf{r}(t)|^{2}\right\rangle$ is the MSD in the $X Y$ plane during the time $t$, starting from the initial time $t_{0}$, and $D$ stands for the lateral diffusion coefficient. Averaging is taken over all molecules and over all initial time $t_{0}$. In figure 6 , the time dependence of the lateral MSD of Paracetamol in the DPPC simulations is shown.

By plotting the logarithm of MSD versus the logarithm of time, the diffusion coefficient can be determined from the $y$-intercept of the tangent to the function where the slope equals 1 (a slope of 1 defines the diffusive regime). As shown in figure 6 (and supplementary data figure S4), the slope was fitted at the time interval 5-45 ns, where the MSD curves behaved linearly. In table 1, the computed lateral diffusion coefficients for Paracetamol and lipids are listed. The lateral diffusion coefficient for reference systems (DPPC and DMPC) are in good agreement with previous simulations. ${ }^{42-44}$ The change in lateral diffusion of Paracetamol molecules in DPPC is much faster compared to DMPC. The reason is the temperature difference of simulations, $323 \mathrm{~K}$ for DPPC and $310 \mathrm{~K}$ for DMPC, and the fact that, the diffusion coefficient is exponentially related to the temperature. The diffusion coefficient of Paracetamol increases by increase in the number of Paracetamol molecules in both DMPC and DPPC, as shown in figure 6 (and supplementary data figure S4). However, the lateral diffusion of both DPPC and DMPC decreases with addition of Paracetamol, since the drug molecules, penetrating in the lipid membrane, occupy some of the free volume spaces in the membrane and as a result decrease the lateral diffusion of the lipids. In a membrane, the free volume fraction is an estimate of the volume fraction that is accessible to a small molecule. The rate of lateral diffusion controls the fluctuations of the free volume, and thus the translational mobility of the lipids is closely connected to the permeability of the small molecules across the membrane. One argument that supports this hypothesis is that the water permeability across lipids is proposed to depend on the lateral diffusion rates of lipids. ${ }^{45}$ Also, the lateral diffusion of the DPPC is higher than that of DMPC in presence of the same number of Paracetamol molecules, which is due to the difference in their area per lipid. The area per lipid in DPPC lipid bilayer has the higher value (see table 1); therefore, it has more free volume compared to DMPC lipid bilayer.

\subsection{Electrostatic potential}

One of the important properties of lipid bilayers is the electrostatic potential across the membrane, which may be relevant to understanding of mechanisms behind the functioning of ion channels. ${ }^{46}$ The membrane electrostatic potential arises due to specific preferential orientations of the lipid head group dipoles and water dipoles at the membrane-water interface. ${ }^{14}$ Hence, it is often referred to as the bilayer dipole potential. Due to translational symmetry in $X$ and $Y$ directions, the electrostatic potential depends only on Z-coordinate. In figure 7 (and supplementary data figure S5), the total electrostatic potentials of the simulated systems are presented. Values for the electrostatic potential in the middle of the membrane $\left(\omega_{\text {mid }}\right)$, as well as the maximum potential values $\left(\omega_{\max }\right)$ are given in table 1 . For reference systems, the results show a positive dipole potential of $500-600 \mathrm{mV}$ in the middle of the bilayer, which is in good agreement with previous simulation results using GROMOS force field. ${ }^{47,48}$ Obtaining accurate 


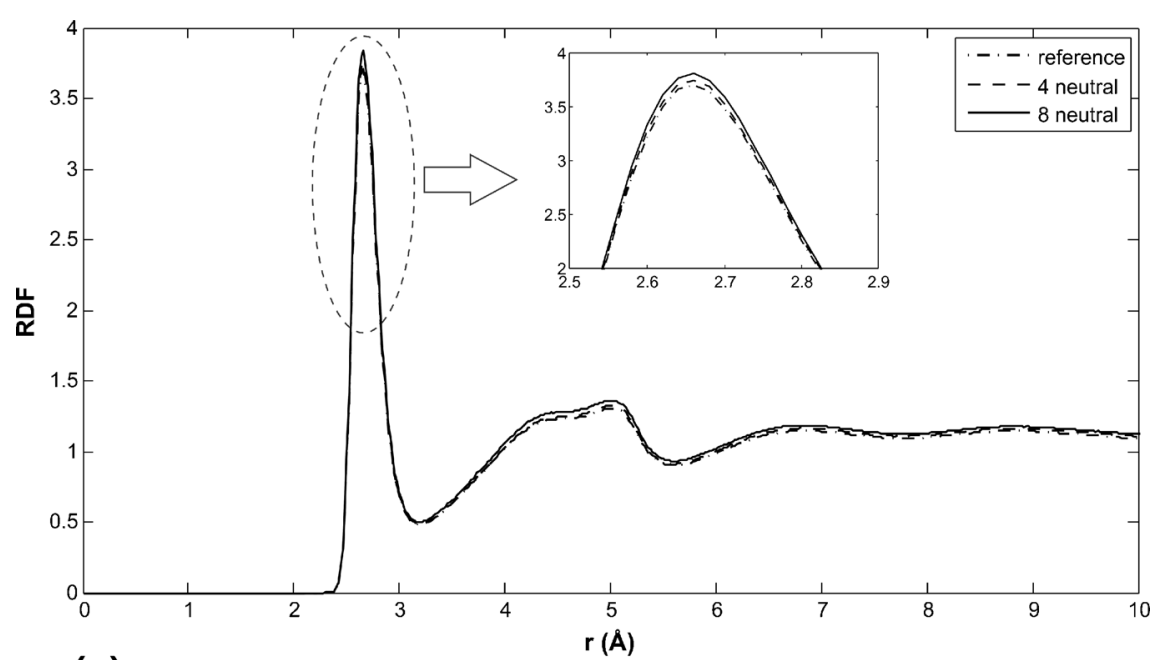

(a)

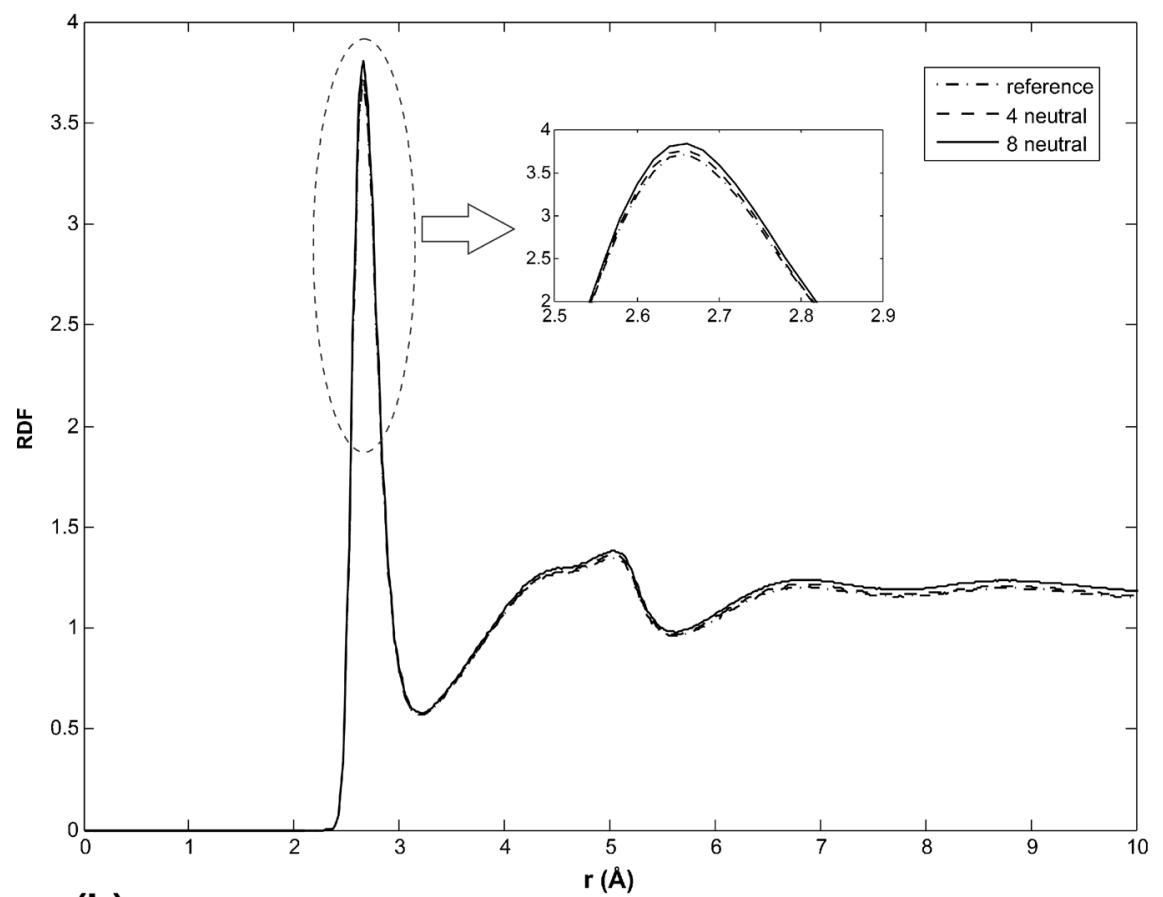

(b)

Figure 5. RDF of water oxygen around phosphate oxygen of DPPC (a) and DMPC (b) lipid bilayers.

experimental determination of the electrostatic potential is difficult and there are different reported values. However, most of the $\left(\omega_{\text {mid }}\right)$ values are in the range of 300-800 $\mathrm{mV} .^{49}$ Other experimental studies have shown a lower value for $\left(\omega_{\text {mid }}\right)$ in the range of $220-280 \mathrm{mV} .^{50}$ From the result obtained, it can be seen that Paracetamol causes a slight decrease of about $10 \mathrm{mV}$ for DPPC and $50 \mathrm{mV}$ for DMPC in the electrostatic potential in their head group regions. As figure 7 indicates, in the hydrocarbon region of the lipid tails in presence of four neutral Paracetamol molecules, the electrostatic potential is almost the same as that of the reference system, whereas the electrostatic potential slightly increases in presence of eight neutral Paracetamol molecules. This increase in electrostatic potential can be attributed to deformation of lipid tails and their higher interactions in the tail region; furthermore, the dipole moments associated with the carbonyl groups and the reorientation of water dipoles in presence of eight molecules cause an increase in the electrostatic potential. ${ }^{14}$

\subsection{Potential of mean force}

Partitioning of the drug and its mean position in the membrane can be described by a free energy $(\Delta G)$ 
Table 3. Coordination numbers in the simulated systems.

\begin{tabular}{lc}
\hline System & Coordination number \\
\hline DPPC reference & 1.29 \\
DMPC reference & 1.22 \\
4 Paracetamol in DPPC & 1.30 \\
8 Paracetamol in DPPC & 1.32 \\
4 Paracetamol in DMPC & 1.26 \\
8 Paracetamol in DMPC & 1.28 \\
\hline
\end{tabular}

profile along the normal to the lipid bilayer membrane, which is also known as PMF. ${ }^{1,51}$ PMF can be calculated by biased MD simulations. Different methods for calculation of free energy profile have been developed, namely umbrella sampling, ${ }^{3,52}$ and $\mathrm{z}$ constraint method. ${ }^{53,54}$ In this study, PMF was calculated by using the umbrella sampling method. In umbrella sampling, a harmonic potential is applied between COMs of Paracetamol and DPPC lipid bilayer. The distance between COMs of Paracetamol and DPPC lipid bilayer was restrained by a harmonic force constant of 500 $\mathrm{KJ} \mathrm{mol}^{-1} \mathrm{~nm}^{-2}$. Then, the free energy profile was calculated by the following equation: ${ }^{55}$

$$
\Delta G(z)=-R T \operatorname{Ln} P(z)+U(z),
$$

where $P(z)$ and $U(z)$ are respectively, the distribution of distance and biasing potential along the bilayer normal. The forces exerted on the Paracetamol were analysed, and the free energy profile was obtained by the weighted histogram analysis method (WHAM, g_wham program). ${ }^{56,57}$ Error estimate of the free energy

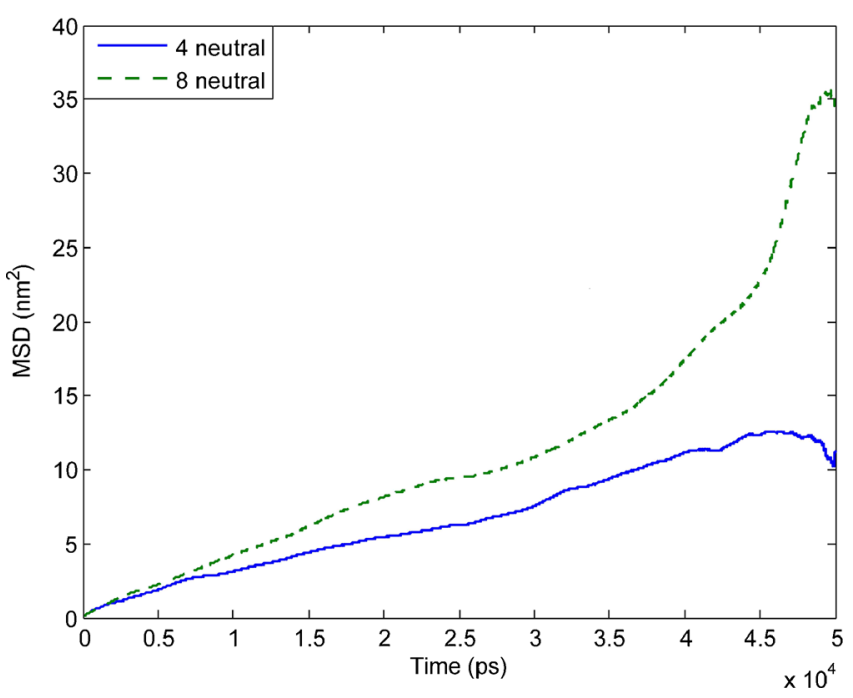

Figure 6. Lateral mean square displacement of Paracetamol in DPPC lipid bilayers.

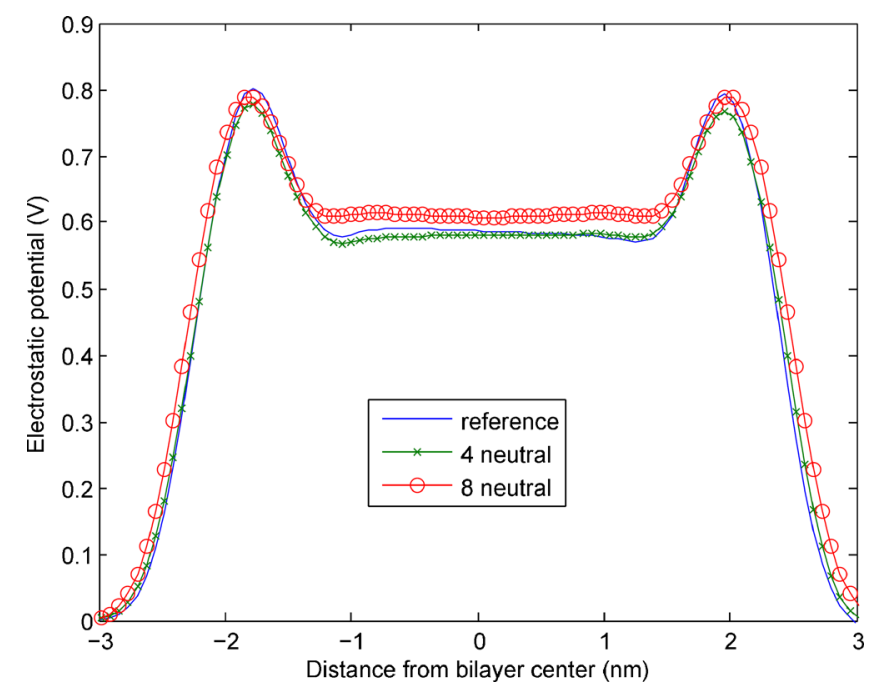

Figure 7. DPPC membrane electrostatic potential.

profile was calculated as integrated standard deviations of the mean calculated over the bins of 50 bootstraps generated by Bayesian bootstrap analysis by the g_wham program. In figure 8 , the free energy profile of Paracetamol in DPPC lipid bilayer is shown. The free energy decreases as Paracetamol enters the lipid bilayer. The first minimum in figure 8 is at $1.817 \mathrm{~nm}$ from the bilayer centre, which is in the thermally accessible region. The thermally accessible region (1.718$1.9 \mathrm{~nm})$, is an area with an energy barrier of $R T$ $\left(0.642 \mathrm{kcal} \mathrm{mol}^{-1}\right.$ at $\left.323 \mathrm{~K}\right)$, which is obtained from the energy minimization in PMF calculations. ${ }^{2}$ As Paracetamol moves deeper into the bilayer, the free energy rises from its minimum to a maximum. Being a polar molecule, going deeper into the centre of the lipid bilayer is less favourable due to the hydrophobic nature

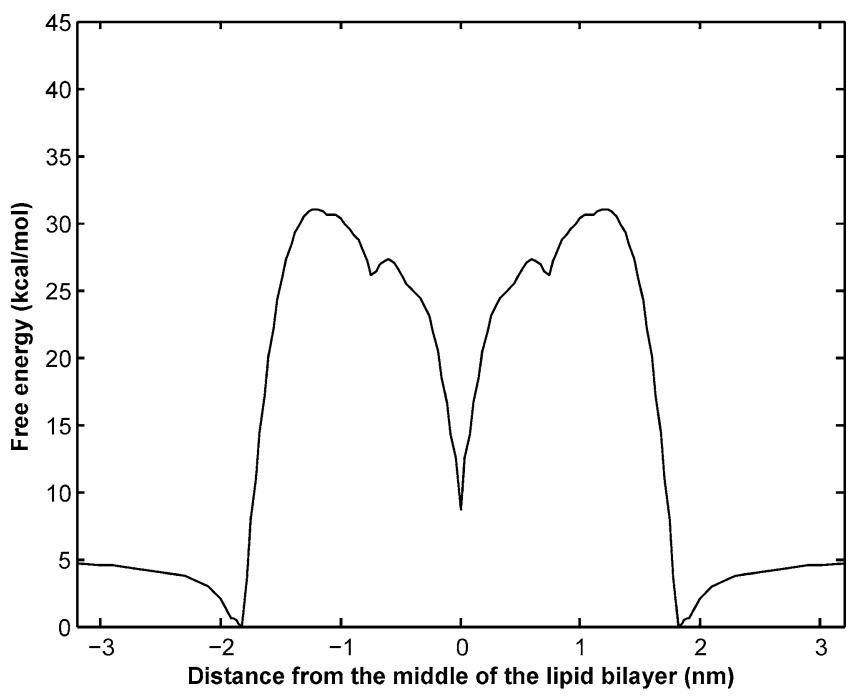

Figure 8. Free energy profile of Paracetamol in DPPC lipid bilayer. 
of the lipid tails at the middle. These factors generate high energy barrier for the permeation process. The bilayer centre barrier $\left(\Delta G^{\mathrm{pen}}=31.01 \pm 0.2 \mathrm{kcal}\right.$ $\mathrm{mol}^{-1}$ ) determines the energy difference between minimum and maximum values of the free energy profile. The free energy profile shows a sharp decrease in the middle of lipid bilayer, which is due to hydrogen bond breaking between the drug and water molecules.

\section{Conclusion}

In this study, the atomistic MD with the united atom model has been utilized to analyse the system of neutral Paracetamol drug molecules in the lipid bilayer membranes of DPPC and DMPC. The biased MD simulations were used to calculate the free energies and to study the different behaviours of neutral Paracetamol drug molecules in the DPPC and DMPC lipid bilayers as well as effect of the drug on membrane properties. Free energy profile of the drug in the lipid bilayer membrane was obtained and used to interpret the drug action. From the results, it can be concluded that neutral form of the Paracetamol shows a generally similar behaviour in DPPC and DMPC lipid bilayers. During simulation, the Paracetamol molecule prefers the head group region of the bilayer (distantly located from the bilayer centre). Neutral form of Paracetamol decreases C-Deuterium order parameters in the lipid tails in DMPC and DPPC. The long axis of Paracetamol adopts an inward orientation pointing to the lipid bilayer membranes. The hydrogen bonding between water molecules and Paracetamol shows similar trends in presence of DMPC and DPPC lipid bilayers. Most of the hydrogen bonds are formed between carbonyl oxygen of the Paracetamol molecules and water. Presence of Paracetamol has a slight effect on hydrogen bonding between lipids and water molecules. RDF of oxygen atom in water around oxygen atom in phosphate of the lipid bilayers shows somewhat higher value, in presence of the Paracetamol, compared to the reference systems. While Paracetamol concentration enhances its lateral diffusion in the lipid bilayer membrane, it causes a decrease in the lateral diffusion of the lipid molecules in the membrane. The most interesting results deduced from this study can be outlined as follows; (i) for the system with four neutral drug molecules, the dipole electrostatic potential inside the membrane has almost the same value as that of the reference system; whereas, for the system with eight neutral drug molecules, the dipole electrostatic potential has higher values than that of the reference; (ii) the variation in concentration of the drug causes a slight change in the electrostatic potential in the head group region of the lipid bilayers; (iii) the free energy profile has its first minimum at $1.817 \mathrm{~nm}$ from the bilayer centre and the bilayer penetration barrier is observed which is due to the hydrogen bonding between the drug and water molecules and (iv) the free energy shows a sharp decrease in the middle of lipid bilayer.

\section{Supplementary information}

The supplementary information figures S1-S4 and table $\mathrm{S} 1$ can be seen in www.ias.ac.in/chemsci website.

\section{Acknowledgements}

We thank the High Performance Computing Research Center (HPCRC) of Amirkabir University of Techno logy (Tehran Polytechnic) for provision of computer facilities.

\section{References}

1. Berka K, Hendrychová T, Anzenbacher P and Otyepka M 2011 J. Phys. Chem. 11511248

2. Paloncýová M, Berka K and Otyepka M 2012 J. Chem. Theory Comput. 81200

3. Boggara M B and Krishnamoorti R 2010 Biophys. J. 98 586

4. Wu N A N, Palczewski K and Mu D J 2008 Pharmacol. Rev. 6043

5. Sneader W 2005 Drug discovery: A history (ed) N J Hoboken (New York: Wiley) p. 439

6. Cormie P J, Nairn M and Welsh J 2008 B M J 3372154

7. Ahmad S R 2007 Lancet 369462

8. Bannwarth B, Netter P, Lapicque F, Gillet P, Pere P, Boccard E, Royer R J and Gaucher A 1992 Br. J. Clin. Pharmacol. 3479

9. Bales J R, Nicholson J K and Sadler P J 1985 Clin. Chem. 31757

10. Prescott L F 1980 Br. J. Clin. Pharmacol. 10291

11. von Bruchlausen F 1982 J. Baumann Life. Sci. 301783

12. Ward B and Alexander-Williams J M 1999 Acute Pain 2 139

13. Steventon G B, Mitchell S C and Waring R H 1996 Drug Metabol. Drug Interact. 13111

14. Mojumdar E H and Lyubartsev A P 2010 Biophys. Chem. 15327

15. Ghadamgahi M and Ajloo D 2013 J. Chem. Sci. 125627

16. Yousefpour A, Amjad S I, Nademi Y and Modarress H 2013 Int. J. Quantum Chem. 1131919

17. Marrink S J, de Vries A H and Tieleman D P 2009 Biochim. Biophys. Acta 1788149

18. Jana B, Pal S and Bagchi B 2012 J. Chem. Sci 124317

19. Tieleman D P and Berendsen H J C 1996 Chem. Phys. J. 1054871

20. Berger O, Edholm O and Jahnig F 1997 Biophys. J. 72 2002 
21. Lindahl E and Edholm O 2000 Biophys. J. 79426

22. Benz R W, Castro-Roman F, Tobias D J and White S H 2005 Biophys. J. 88805

23. Hogberg C J and Lyubartsev A P 2006 J. Phys. Chem. B 11014326

24. Berendsen H J C, Postma J P M, van Gunsteren W F and Hermans J 1981 Intermolecular forces (eds) B Pullman, (Reidel, Dordrecht ) p. 331

25. Schuttelkopf A W and van Aalten D M 2004 Acta Crystallogr. Sec. D: Biol. Crystallogr. 601355

26. Hess B, Kutzner C, Van Der Spoel D and Lindahl E 2008 J. Chem. Theory Comput. 4435

27. Bussi G, Donadio D and Parrinello M 2007 J. Chem. Phys. 126014101

28. Hess B, Bekker H, Berendsen H J C and Fraaije J G E M 1997 Comput. Chem. J. 181463

29. Parrinello M and Rahman A 1980 Phys. Rev. Lett. 45 1196

30. Darden T, York D and Pedersen L 1993 Chem. Phys. J. 9810089

31. Snyman J A 2005 Practical mathematical optimization: An introduction to basic optimization theory and classical and new gradient-based algorithms (New York: Springer) p. 40

32. Oldfield E, Chapman D and Derbyshire W 1971 FEBS Lett. 16102

33. Petrache H I, Dodd S W and Brown M F 2000 Biophys. J. 793172

34. Seelig A and Seelig J 1974 Biochemistry 134839

35. Porasso R D, Bennett W F, Oliveira-Costa S D and Lopez Cascales J J 2009 J. Phys. Chem. B 1139988

36. van der Spoel D, van Maaren P J, Larsson P and Timneanu N 2006 J. Phys. Chem. B 1104393

37. Modig K, Pfrommer B G and Halle B 2003 Phys. Rev. Lett. 90075502
38. Nagle J F 1993 Biophys. J. 641476

39. Feller S E, Venable R M and Pastor R W 1997 Langmuir 136555

40. Song Y, Guallar V and Baker N A 2005 Biochemistry 4413425

41. Venable R M, Zhang Y, Hardy B J and Pastor R W 1993 Science 262223

42. Patra M, Karttunen M, Hyvönen M T, Falck E and Vattulainen I 2004 J. Phys. Chem. B 1084485

43. Hogberg C J and Lyubartsev A P 2006 J. Phys. Chem. B 11014326

44. Wohlert J J and Edholm O 2006 J. Chem. Phys. 125 204703

45. Haines T H 1994 FEBS Lett. 346115

46. Hogberg C J and Lyubartsev A P 2008 Biophys. J. 94 525

47. Patra M, Salonen E, Terama E, Vattulainen I, Faller R, Lee BW, Holopainen J and Karttunen M 2006 Biophys. J. 901121

48. Wohlert J and Edholm O 2004 Biophys. J. 872433

49. Cevc G 1990 Biochim. Biophys. Acta 1031311

50. Clarke R J 2001 Adv. Colloid Interface Sci. 89-90 263

51. Neale C, Bennett W F D, Tieleman D P and Pomès R 2011 J. Chem. Theory Comput. 74175

52. MacCallum J L and Tieleman D P 2006 J. Am. Chem. Soc. 128125

53. Orsi M and Essex J W 2010 Soft Matter 63797

54. Orsi M, Sanderson W E and Essex J W 2009 J. Phys. Chem. B 11312019

55. Xiang T-X and Anderson B D 2006 Adv. Drug Delivery Rev. 581357

56. Kumar S, Rosenberg J, Bouzida D, Swensen R H and Kollman P A 1992 J. Comput. Chem. 131011

57. Hub J S, Groot B L D and Spoel D V D 2010 J. Chem. Theory Comput. 63713 